\title{
Primary Tumor Resection in Stage IV Breast Cancer: Consistent Benefit, or Consistent Bias?
}

\author{
Seema A. Khan, MD \\ Department of Surgery and the Robert H Lurie Cancer Center, Feinberg School of Medicine of Northwestern University, \\ 675 North Saint Clair, Galter 13-174, Chicago, IL 60611, USA
}

The recommended primary treatment approach for women with metastatic breast cancer and an intact primary tumor is the use of systemic therapy, with local therapy for the primary tumor reserved for palliation of symptoms. The past 6 years have seen an accelerating pace of publication of studies examining survival outcomes relative to the surgical resection of the intact primary tumor in women with metastatic breast cancer. ${ }^{1-4}$ These show that in fact about half the women presenting with de novo metastatic disease undergo resection of the primary tumor and suggest that women so treated survive longer than those treated without resection. In analyses that adjust for tumor burden (number of metastatic sites), types of metastases (visceral, nonvisceral), and the use of systemic therapy, the hazard of death is reduced by $40-50 \%$ in women receiving surgical treatment of the primary tumor. Thus, five retrospective studies including the report by Fields et al. ${ }^{5}$ in the present issue of the Annals of Surgical Oncology, present us with consistent evidence that either surgical therapy of the primary tumor has a substantial survival benefit in women with metastatic breast cancer, or there is a strong and consistent selection bias driving the use of surgery in women who have more favorable profiles (i.e., younger age, smaller tumor burden, better access to care). It is also pos-

Received June 18, 2007; accepted June 20, 2007; published online: September 22, 2007.

Address correspondence and reprint requests to: Seema A. Khan, MD; E-mail: skhan@nmh.org

Published by Springer Science+Business Media, LLC ๔ 2007 The Society of Surgical Oncology, Inc. sible that surgery is a surrogate indicator of more aggressive therapy overall, including more aggressive systemic therapy, which translates into better survival. All authors acknowledge the problem of selection bias in the interpretation of these data, and all advocate for a randomized trial to settle this question.

In this setting, what can we learn from additional single-institution retrospective series addressing this question? Single-institution studies are necessarily smaller than the large data sets that are available from the National Cancer Database $(\mathrm{NCDB})^{1}$ and the Surveillance, Epidemiology, and End-Results (SEER) program, ${ }^{4}$ but provide richer detail regarding the specifics of local and systemic therapy and the course of the local disease. Such detailed information can garner data on trends and practices to guide the design of a randomized trial. Local therapy questions that require further definition include the timing of surgery, the importance of free surgical margins, and the benefit of axillary clearance and radiotherapy. Additionally, data from single-institution series may generate hypotheses regarding subsets of patients who are more likely to benefit from local therapy.

The timing of surgery (early, after response to systemic therapy or later, only if indicated for palliation) has not been examined in any detail, although large databases such as NCDB and SEER capture the first course of treatment most accurately, and therefore women reported as having had surgical therapy would most likely have received this early in their course. This issue is relevant to the hypothesis that local therapy of the primary tumor is beneficial for 
women with metastatic disease: If the tumor functions as a source of new metastatic deposits, treating it early in the course would logically have greater benefit.

Despite the burgeoning data on surgical resection of the primary tumor, information on the use of axillary dissection in this population is scant. So far, it has been assumed that women with stage IV disease are likely to die before axillary control becomes a problem. However if surgical extirpation of the primary tumor is beneficial, it seems implausible that therapy for axillary disease would not add benefit, and one previous study suggests a trend toward a larger benefit for women who had both negative surgical margins and axillary dissection (HR 0.2, 95\% CI 0.02-1.9). ${ }^{2}$

Similarly, regional radiotherapy data are limited to the study of Rapiti et al., who reported that local radiation was used in women undergoing breastconserving surgery, and the lack of radiotherapy increased the hazard of death independently. ${ }^{2}$ The inability to distinguish local radiation from radiation to metastatic sites is a limitation of the data in the $\mathrm{NCDB},{ }^{1}$ and in the SEER ${ }^{4}$ program. This is an area where single-institution reviews could provide insights that would inform both trial design and (in the interim) patient care.

The published data are unanimous in identifying smaller tumor size as a selection factor for surgical therapy. It appears unlikely that the resection of T1-2 tumors is motivated by a need to palliate symptoms and suggests that uncontrolled local disease is a feared complication among both physicians and patients. It is reasonable to assume that complete resection is protective against uncontrolled chest wall disease, but direct data are limited. A retrospective study of 111 women, presented in abstract form at the Society of Surgical Oncology in 2007, suggests not only that surgery is strongly protective against uncontrolled chest wall disease, but also that a controlled chest wall mediates the benefit of surgical resection. ${ }^{6}$

Looking at the report from Fields et al. in this context, we again find confirmation that surgical resection of the primary tumor is currently performed in about half the women presenting with stage IV disease and is associated with an approximate halving of the hazard of death during the follow-up period in multivariate analyses. ${ }^{5}$ As in previous studies, the use of surgery is associated with smaller tumor size. ${ }^{1,2,4}$ The surgical group in this study included patients who underwent surgical therapy at any point in their course, and in about half these women surgery was undertaken to palliate symptoms. It would be of great interest to know how frequently surgical therapy for palliation was successful in maintaining a disease-free chest wall (particularly if radiotherapy was not used). Axillary evaluation was performed on 143 of 187 women (76\%), but no further information is presented, and it is unclear if and how these data were included in the multivariate survival analysis. There is no discussion of radiotherapy data.

Fields et al. find that women with bone-only metastases lived longer than those with metastases at other sites, with an adjusted hazard ratio of 0.76 (95\% CI $0.58-0.98) .{ }^{5}$ These results are consistent with the known indolent course of osseous metastases. Thus, women with bone-only disease may benefit from surgical resection of the primary because they have a longer period of time during which they can develop uncontrolled chest wall disease; but in the absence of randomized data, we cannot conclude that these women derive a greater survival benefit from surgery. Finally, Fields et al. have included a comorbidity score (ACE-27) that categorizes comorbid conditions as none, mild, moderate, and severe; they find that this score is not significantly predictive of survival. ${ }^{5}$ Earlier data from the same institution showed that the ACE-27 had the greatest impact on survival when the force of mortality of the malignant diagnosis was small (e.g., early breast or prostate cancer) and least when it was high (e.g., stage IV breast cancer) ${ }^{7}$. Thus, in a highly lethal disease such as stage IV breast cancer, comorbidity does not seem to play a significant role in determining survival.

Despite these continued attempts to adjust for additional parameters in analysis, the fact remains that no study will fully account for the selection biases that confound the interpretation of retrospective data; and that a consistent, reproducible bias will show up in all analyses that are subject to that bias. The question of whether or not local therapy matters in stage IV disease is increasingly important, given the documented prolongation in survival of women with stage IV breast cancer, ${ }^{8}$ and the anticipation that survival for this group will continue to improve with newer targeted systemic agents. In addition, this question has wider implications than the specific population of women who present with de novo metastases. An analogous situation exists in women with synchronous in-breast recurrence and distant metastases, and ultimately the answer to this question will have implications for women with oligometastases that may be suitable for local therapy.

The design of a prospective study will need to address the full gamut of local therapy for the primary tumor: surgical resection with free margins, axillary control, and radiotherapy. Even if one were to 
concede that it is a simple matter to recommend surgical extirpation of the primary tumor without the expense and effort of a randomized trial, axillary and radiation therapy pose economic and personal burdens that cannot be justified based on the available data. A randomized trial is required.

Arguments against a randomized trial include questions as to whether limited cooperative group resources should be devoted to a small minority of patients, whether randomization will be accepted by patients and physicians, and whether systemic therapy can be standardized sufficiently in a heterogeneous patient population. However, given the apparent large effect of surgery, it appears unlikely that specific systemic therapy regimens will have a large enough impact on survival to obscure the effects of surgery. Randomization is less likely to be a barrier for physicians and patients if axillary dissection and radiotherapy are part of the question, since these interventions are not widely used in this group of patients at present. Preliminary sample size calculations based on a $10 \%$ improvement in 3-year survival suggest that such a trial would require about 700 patients, and if adopted across cooperative groups, could be completed in 4 years if $2 \%$ of eligible women participated. A similar trial by Flanigan et al. took 6 years to complete, but provided invaluable data for patients with advanced renal cell carcinoma. ${ }^{9}$

We are faced with the possibility of changing the paradigm that governs our concepts of the interplay of a primary tumor and metastases and the treatment strategies that result from these concepts. A randomized trial, with incorporation of exciting correlative science questions, has enormous potential rewards for our patients.

\section{REFERENCES}

1. Khan SA, Stewart AK, Morrow M. Does aggressive local therapy improve survival in metastatic breast cancer? Surgery 2002; 132:620-6.

2. Rapiti E, Verkooijen HM, Vlastos G, Fioretta G, NeyroudCaspar I, Sappino AP, Chappuis PO, Bouchardy C. Complete excision of primary breast tumor improves survival of patients with metastatic breast cancer at diagnosis. J Clin Oncol 2006; 24:2743-9.

3. Babiera GV, Rao R, Feng L, Meric-Bernstam F, Kuerer HM, Singletary SE, Hunt KK, Ross MI, Gwyn KM, Feig BW, Ames FC, Hortobagyi GN. Effect of primary tumor extirpation in breast cancer patients who present with stage IV disease and an intact primary tumor. Ann Surg Oncol 2006; 13:776-82.

4. Gnerlich J, Jeffe DB, Deshpande AD, Beers C, Zander C, Margenthaler JA. Surgical Removal of the Primary Tumor Increases Overall Survival in Patients With Metastatic Breast Cancer: Analysis of the 1988-2003 SEER Data. Ann Surg Oncol 2007; DOI 10.1245/s10434-007-9438-0 [Online May 24, 2007].

5. Fields RC, Jeffe DB, Trinkaus K, Zhang Q, Carey A, Aft R, Dietz JR, Eberlein TJ, Gillanders WE, Margenthaler JA. Surgical resection of the primary tumor is associated with increased long-term survival in patients with Stage IV breast cancer after controlling for site of metastasis. Ann Surg Oncol 2007; 14; doi: 10.1245/s10434-007-9527-0 [Online August 9, 2007].

6. Hazard HW, Gorla SR, Kim J, Scholtens D, Gradishar WJ, Khan SA. Surgical resection of the primary tumor in stage IV breast cancer and survival. Presented at the Society of Surgical Oncology (SSO) 60th Annual Cancer Symposium 2007, vol 14 (suppl), p 84, abstract P174.

7. Read WL, Tierney RM, Page NC, Costas I, Govindan R, Spitznagel EL, Piccirillo JF. Differential prognostic impact of comorbidity. J Clin Oncol 2004; 22:3099-103.

8. Andre F, Slimane K, Bachelot T, Dunant A, Namer M, Barrelier A, Kabbaj O, Spano JP, Marsiglia H, Rouzier R, Delaloge S, Spielmann M. Breast cancer with synchronous metastases: trends in survival during a 14 -year period. J Clin Oncol 2004; 22:3302-8.

9. Flanigan RC, Salmon SE, Blumenstein BA, Bearman SI, Roy V, McGrath PC, Caton JR Jr., Munshi N, Crawford ED. Nephrectomy followed by interferon alfa- $2 \mathrm{~b}$ compared with interferon alfa-2b alone for metastatic renal-cell cancer. $N$ Engl $J$ Med 2001; 345:1655-9. 